\title{
Multiplicative Isometries and Isometric Zero-Divisors
}

\author{
Alexandru Aleman, Peter Duren, María J. Martín, and Dragan Vukotić
}

Abstract. For some Banach spaces of analytic functions in the unit disk (weighted Bergman spaces, Bloch space, Dirichlet-type spaces), the isometric pointwise multipliers are found to be unimodular constants. As a consequence, it is shown that none of those spaces have isometric zero-divisors. Isometric coefficient multipliers are also investigated.

A key result in the theory of Hardy spaces is the classical theorem of F. Riesz to the effect that the zeros of any function in $H^{p}$ can be divided out by a Blaschke product to produce a nonvanishing function with the same $H^{p}$ norm. In other words, the Blaschke products serve as isometric zero-divisors. The theorem is enormously useful because it transfers questions about $H^{p}$ functions to the more tractable space $H^{2}$. More recently, it was shown $[7,9]$ that the Bergman space $A^{p}$ has no isometric zerodivisors. The proof consisted of producing contractive zero-divisors of unit norm, proving their uniqueness up to rotation, and showing that they are not isometric.

In this note we offer, among other results, a much simpler proof that the Bergman spaces have no isometric zero-divisors. This is deduced from the easily proved fact that the only isometric pointwise multipliers of $A^{p}$ are the unimodular constants. With greater effort, the same approach is applied to the Bloch space, the Dirichlet space, and more general spaces with weighted integral norms. It is shown that none of those spaces admit isometric zero-divisors.

The paper concludes with an analogous study of isometric coefficient multipliers in Hardy and Bergman spaces.

\section{Pointwise Multipliers}

Suppose $X$ and $Y$ are Banach spaces of analytic functions in the unit disk $\mathbb{D}$ ). A function $G$ analytic in $\mathbb{D}$ ) is said to be a pointwise multiplier of $X$ into $Y$ if $G f \in Y$ for every $f \in X$. Then the pointwise multiplication operator $M_{G}: X \rightarrow Y$ is defined by $M_{G} f=G f$ for all $f \in X$. Under the assumption that each point-evaluation functional $\phi_{\zeta}(f)=f(\zeta)$ is bounded on $X$, the closed graph theorem shows that $M_{G}$ is

Received by the editors November 29, 2007.

Published electronically June 18, 2010.

The research of the second, third, and fourth authors is supported by MICINN grant MTM200914694-C02-01, Spain. The third and fourth authors are partially supported by the Thematic Network MTM2008-02829-E from MICINN. The fourth author is also partially supported by the European Science Foundation Network HCAA ("Harmonic and Complex Analysis and Applications").

AMS subject classification: 30H05, 46E15.

Keywords: Banach spaces of analytic functions, Hardy spaces, Bergman spaces, Bloch space, Dirichlet space, Dirichlet-type spaces, pointwise multipliers, coefficient multipliers, isometries, isometric zerodivisors. 
a bounded operator. Also, in the nontrivial case where $X \neq\{0\}$, it can be said that $\left\|\phi_{\zeta}\right\|>0$ except perhaps for a discrete set of points $\zeta$ in $\mathbb{D}$ ). The following lemma is well known, but we include a proof for the sake of completeness.

Lemma 1.1 Let X be a nontrivial Banach space of functions analytic in the unit disk, and suppose that each point-evaluation functional is bounded on $X$. If an analytic function $G$ is a pointwise multiplier of $X$ into itself, then $G \in H^{\infty}$ and $\|G\|_{\infty} \leq\left\|M_{G}\right\|$.

Here $H^{\infty}$ denotes the Banach space of bounded analytic functions in the disk, with norm $\|G\|_{\infty}=\sup _{z \in \mathbb{D}}|G(z)|$.

Proof of Lemma 1.1 For each $\zeta \in \mathbb{D}$ ) and $f \in X$,

$$
\begin{aligned}
\left|\phi_{\zeta}(f)\right||G(\zeta)| & =|G(\zeta) f(\zeta)|=\left|\phi_{\zeta}\left(M_{G} f\right)\right| \\
& \leq\left\|\phi_{\zeta}\right\|\left\|M_{G} f\right\|_{X} \leq\left\|\phi_{\zeta}\right\|\left\|M_{G}\right\|\|f\|_{X} .
\end{aligned}
$$

Taking the supremum over all functions $f \in X$ of unit norm, we conclude that $\left\|\phi_{\zeta}\right\||G(\zeta)| \leq\left\|\phi_{\zeta}\right\|\left\|M_{G}\right\|$, so that $|G(\zeta)| \leq\left\|M_{G}\right\|$ wherever $\left\|\phi_{\zeta}\right\|>0$. But the exceptional set where $\left\|\phi_{\zeta}\right\|=0$ is discrete, so the inequality $|G(\zeta)| \leq\left\|M_{G}\right\|$ extends to all $\zeta \in \mathbb{D}$ ).

It is worth remarking that if $X$ is a Banach space of functions analytic in a given domain $\Omega \subset \mathbb{C}$, and if the point-evaluation functional $\phi_{\zeta}$ is bounded on $X$ for each $\zeta \in \Omega$, then the point-evaluations are uniformly bounded on each compact subset of $\Omega$. The proof is a simple application of the principle of uniform boundedness. Indeed, for any compact set $K \subset \Omega$,

$$
\sup _{\zeta \in K}\left|\phi_{\zeta}(f)\right|=\sup _{\zeta \in K}|f(\zeta)|<\infty
$$

for each $f \in X$, so that $\sup _{\zeta \in K}\left\|\phi_{\zeta}\right\|<\infty$.

\section{Hardy and Bergman Spaces}

For $1 \leq p<\infty$, the Hardy space $H^{p}$ consists of the functions $f$ analytic in the unit disk for which

$$
\|f\|_{H^{p}}=\sup _{0 \leq r<1}\left\{\frac{1}{2 \pi} \int_{0}^{2 \pi}\left|f\left(r e^{i \theta}\right)\right|^{p} d \theta\right\}^{1 / p}<\infty .
$$

Under this norm, $H^{p}$ is a Banach space and all point-evaluation functionals are bounded, in view of the estimate

$$
\left.|f(z)| \leq \frac{\|f\|_{H^{p}}}{\left(1-|z|^{2}\right)^{1 / p}}, \quad z \in \mathbb{D}\right) .
$$

See [5] for this and other basic properties of Hardy spaces. 
The Bergman space $A^{p}$ consists of the analytic functions in the disk for which

$$
\|f\|_{A^{p}}=\left\{\int_{\mathbb{D})}|f(z)|^{p} d A(z)\right\}^{1 / p}<\infty,
$$

where $d A(z)=\pi^{-1} d x d y$ is normalized area measure in the disk, with $\left.A(\mathbb{D})\right)=1$. Then $A^{p}$ is a Banach space for $1 \leq p<\infty$, and the estimate

$$
\left.|f(z)| \leq \frac{\|f\|_{A^{p}}}{\left(1-|z|^{2}\right)^{2 / p}}, \quad z \in \mathbb{D}\right),
$$

shows that point-evaluation functionals are bounded. More generally, if $w$ is a nonnegative integrable function on D), the weighted Bergman space $A_{w}^{p}$ is the linear space of analytic functions with finite weighted norm

$$
\|f\|_{A_{w}^{p}}=\left\{\int_{\mathbb{D})}|f(z)|^{p} w(z) d A(z)\right\}^{1 / p} .
$$

Although it is a subspace of the complete space $L^{p}(w d A)$, the weighted Bergman space $A_{w}^{p}$ need not be complete. For instance, it can be shown that $A_{w}^{p}$ is not complete if $w(z)=0$ on some annulus $R<|z|<1$. It is an open problem to describe the weight functions $w$ for which $A_{w}^{p}$ is complete. However, the standard radial weights

$$
w_{\alpha}(z)=(\alpha+1)\left(1-|z|^{2}\right)^{\alpha}, \quad \alpha>-1,
$$

always yield complete Bergman spaces $A_{w_{\alpha}}^{p}$, denoted by $A_{\alpha}^{p}$ for brevity. The pointevaluation functionals are bounded in $A_{\alpha}^{p}$, by the generalized estimate

$$
\left.|f(z)| \leq \frac{\|f\|_{A_{\alpha}^{p}}}{\left(1-|z|^{2}\right)^{(2+\alpha) / p}}, \quad z \in \mathbb{D}\right) .
$$

If $w$ is a weight function for which the point-evaluation functionals are uniformly bounded on compact subsets of the disk, then it can be shown that $A_{w}^{p}$ is a complete space. For a proof, compare the discussion in Section 1.1 of [6], where the proof of completeness is carried out only for the unweighted spaces $A^{p}$.

It is an important property of the Hardy spaces $H^{p}$ and the Bergman spaces $A^{p}$ that the polynomials are dense. In other words, every function in $H^{p}$ or in $A^{p}$ can be approximated in norm by a polynomial. (See $[5,6]$ for proofs.) The same is true in the spaces $A_{\alpha}^{p}$ with standard weights ( $c f$. [6, Section 2.3]), but need not be true in more general weighted Bergman spaces $A_{w}^{p}$.

We now describe the isometric pointwise multipliers of $H^{p}$ and $A_{w}^{p}$. These are the analytic functions $G$ with the property that $\|G f\|=\|f\|$ for all functions $f$ in the given space. It is not necessary to assume that $A_{w}^{P}$ is complete, but we will require the weight function $w$ to be chosen so that every point-evaluation functional is bounded. In order to avoid trivial complications, we will also require that $w(z)>0$ on some open subset of the disk. 


\section{Theorem 2.1}

(i) Let $A_{w}^{p}$ be a weighted Bergman space where the point-evaluation functionals are bounded, and suppose that $w(z)>0$ on some open subset of $1 \mathrm{D})$. Then the only isometric pointwise multipliers of $A_{w}^{p}$ are the unimodular constants.

(ii) The only isometric pointwise multipliers of the Hardy space $H^{p}$ are the inner functions.

Proof In either case, if an analytic function $G$ is an isometric multiplier, then the operator $M_{G}$ is an isometry, so that $\left\|M_{G}\right\|=1$, and Lemma 1.1 tells us that $\|G\|_{\infty} \leq 1$.

(i) For the weighted Bergman space $A_{w}^{p}$ we have

$$
0=\|1\|_{A_{w}^{p}}^{p}-\|G\|_{A_{w}^{p}}^{p}=\int_{\mathbb{D})}\left(1-|G(z)|^{p}\right) w(z) d A(z) .
$$

But Lemma 1.1 says that $1-|G(z)|^{p} \geq 0$ in $\mathbb{D}$ ), and $w(z)>0$ on an open set $E \subset \mathbb{D D})$, so it follows that $1-|G(z)|^{p} \equiv 0$ on $E$. Then since $G$ is analytic, it must be a unimodular constant.

(ii) A similar argument shows that $|G(z)| \leq 1$ in $\mathbb{D}$ ) and $|G(z)|=1$ almost everywhere on $\partial \mathrm{D}$ ), hence $G$ is an inner function.

\section{The Bloch Space}

The Bloch space $\mathcal{B}$ is the Banach space of analytic functions in $1 \mathrm{D})$ with bounded invariant derivative, equipped with the norm

$$
\|f\|_{\mathcal{B}}=|f(0)|+\sup _{z \in \mathbb{D})}\left(1-|z|^{2}\right)\left|f^{\prime}(z)\right| .
$$

It is a large space in the sense that it is not separable; in particular, the polynomials are not dense in $\mathcal{B}$. The closure of the polynomials in the Bloch norm turns out to be the little Bloch space $\mathcal{B}_{0}$ defined by the condition

$$
\lim _{|z| \rightarrow 1}\left(1-|z|^{2}\right)\left|f^{\prime}(z)\right|=0
$$

The spaces $\mathcal{B}$ and $\mathcal{B}_{0}$ are intimately connected with the Bergman space $A^{1}$. The dual space of $\mathcal{B}_{0}$ can be identified with $A^{1}$, and the dual of $A^{1}$ is $\mathcal{B}$. Every function $f$ in $\mathcal{B}$ satisfies the growth condition

$$
\left.|f(z)| \leq\left(1+\log \frac{1}{1-|z|}\right)\|f\|_{\mathcal{B}}, \quad z \in \mathbb{D}\right)
$$

which shows that point-evaluation functionals are bounded in $\mathcal{B}$ and therefore in $\mathcal{B}_{0}$. See for instance [6] for properties of the Bloch space.

Brown and Shields [1], among others, found a description of the pointwise multipliers of the Bloch space into itself. Cima and Wogen [3] described the surjective isometries. The following theorem shows, however, that very few pointwise multipliers act isometrically. 
Theorem 3.1 The only isometric pointwise multipliers of the Bloch space $\mathcal{B}$ are the unimodular constants. The same is true for the little Bloch space $\mathcal{B}_{0}$.

Proof If an analytic function $G$ is an isometric multiplier of the Bloch space $\mathcal{B}$, we conclude from Lemma 1.1 that $|G(z)| \leq 1$ in $\mathbb{D}$ ). The Schwarz-Pick lemma then applies to show that

$$
\left.\left(1-|z|^{2}\right)\left|G^{\prime}(z)\right| \leq 1-|G(z)|^{2}, \quad z \in \mathbb{D}\right) .
$$

Since $M_{G}$ is an isometry of $\mathcal{B}$, we have $\|G\|_{\mathcal{B}}=\|1\|_{\mathcal{B}}=1$ and inductively $\left\|G^{n}\right\|_{\mathcal{B}}=$ 1 for $n=1,2, \ldots$. This means that

$$
|G(0)|^{n}+\sup _{z \in \mathbb{D}}\left(1-|z|^{2}\right) n|G(z)|^{n-1}\left|G^{\prime}(z)\right|=1
$$

for all $n$. Invoking the Schwarz-Pick lemma, we infer that

$$
1 \leq|G(0)|^{n}+\sup _{z \in \mathbb{D}} n|G(z)|^{n-1}\left(1-|G(z)|^{2}\right) .
$$

However, elementary calculus shows that the function $u_{n}(r)=n r^{n-1}\left(1-r^{2}\right)$ attains its maximum value in the interval $[0,1]$ at the point $r_{n}=\sqrt{(n-1) /(n+1)}$. Thus

$$
u_{n}(r) \leq u_{n}\left(r_{n}\right)=\frac{2 n}{n+1}\left(1-\frac{2}{n+1}\right)^{(n-1) / 2} .
$$

As $n \rightarrow \infty$, this last quantity tends to $2 / e<3 / 4$. This shows that $u_{n}(r)<3 / 4$ for all $r \in[0,1]$ when $n$ is sufficiently large. It then follows from (3.1) that $|G(0)|^{n}>1 / 4$ for large $n$, so that $|G(0)| \geq 1$. Since $|G(z)| \leq 1$ in $\mathbb{D})$, the conclusion is that $|G(0)|=1$ and so $G(z)$ is a constant of modulus one. The proof applies verbatim to the little Bloch space $\mathcal{B}_{0}$, since the norm is the same.

\section{Dirichlet-Type Spaces}

The Dirichlet space $\mathcal{D}$ is the Hilbert space of all analytic functions in $1 \mathrm{D})$ with finite Dirichlet integral, endowed with the norm given by

$$
\|f\|_{\mathcal{D}}^{2}=|f(0)|^{2}+\int_{\mathbb{D}}\left|f^{\prime}(z)\right|^{2} d A(z)=\left|a_{0}\right|^{2}+\sum_{n=1}^{\infty} n\left|a_{n}\right|^{2},
$$

where $f(z)=\sum_{n=0}^{\infty} a_{n} z^{n}$. The inequality

$$
|f(z)| \leq\left(1+\sqrt{\log \frac{1}{1-|z|^{2}}}\right)\|f\|_{\mathcal{D}}
$$

shows that point-evaluation functionals are bounded. An analytic function $f$ belongs to the Dirichlet space if and only if $f^{\prime}$ is in the Bergman space $A^{2}$.

Because $\mathcal{D}$ is a Hilbert space, it has many isometries. Stegenga [14] has characterized the pointwise multipliers of the Dirichlet space into itself. Again we observe that very few of them are isometric. 
Theorem 4.1 The only isometric pointwise multipliers of the Dirichlet space $\mathcal{D}$ are the unimodular constants.

Proof It is easy to see that the Dirichlet space is invariant under multiplication by polynomials. Suppose that $G(z)=\sum_{n=0}^{\infty} a_{n} z^{n}$ is an isometric multiplier of $\mathcal{D}$. Then $\|G\|_{\mathcal{D}}=\|1\|_{\mathcal{D}}=1$, so that

$$
\left|a_{0}\right|^{2}+\sum_{n=1}^{\infty} n\left|a_{n}\right|^{2}=1
$$

In similar fashion, the relation $\|z G\|_{\mathcal{D}}=\|z\|_{\mathcal{D}}=1$ says that

$$
\sum_{n=0}^{\infty}(n+1)\left|a_{n}\right|^{2}=1 \text {. }
$$

Subtracting the first equation from the second, we conclude that $\sum_{n=1}^{\infty}\left|a_{n}\right|^{2}=0$, so that $G(z) \equiv a_{0}$. Since $\|G\|_{\mathcal{D}}=1$, it is clear that $\left|a_{0}\right|=1$.

We now turn to a broad generalization of Theorem 4.1 whose proof will be more complicated. For $1 \leq p<\infty$, we define the Dirichlet-type space $D_{w}^{p}$ to consist of all analytic functions in the unit disk with finite norm

$$
\|f\|_{D_{w}^{p}}=\left\{|f(0)|^{p}+\int_{\mathbb{D})}\left|f^{\prime}(z)\right|^{p} w(z) d A(z)\right\}^{1 / p} .
$$

Here the weight function $w$ is any nonnegative integrable function. Special choices of the weight function lead to some familiar spaces as special cases. If $w(z)=$ $(p-1)\left(1-|z|^{2}\right)^{p-2}$ then $D_{w}^{p}$ is the analytic Besov space $B^{p}$ of all analytic functions whose invariant derivative belongs to the $L^{p}$ space with respect to hyperbolic area measure. The choice $w(z)=\left(1-|z|^{2}\right)^{p}$ gives the Bergman space $A^{p}$ with an equivalent but different norm. When $p=2$ the space $D_{w}^{2}$ is called a weighted Dirichlet space, but it need not be complete. The weight function $w(z)=-2 \log |z|$ is of particular interest, in view of the Littlewood-Paley formula

$$
2 \int_{\mathbb{D})}\left|f^{\prime}(z)\right|^{2} \log \frac{1}{|z|} d A(z)=\sum_{n=1}^{\infty}\left|a_{n}\right|^{2}
$$

where $f(z)=\sum_{n=0}^{\infty} a_{n} z^{n}$. With this choice of weight function, the space $D_{w}^{2}$ is none other than the Hardy space $H^{2}$, with precisely the same norm.

The next theorem says that only in this last situation does the Dirichlet-type space $D_{w}^{p}$ have nontrivial isometric multipliers.

Theorem 4.2 Let $1 \leq p<\infty$. Suppose that the Dirichlet-type space $D_{w}^{p}$ is complete and that point-evaluations are bounded. Then $D_{w}^{p}$ has nonconstant isometric pointwise multipliers if and only if $p=2$ and $w(z)=-2 \log |z|$ a.e. in $\mathrm{DD}$. In this case $D_{w}^{p}=H^{2}$ and the isometric multipliers are precisely the inner functions. 
Proof If an analytic function $G$ is an isometric multiplier of $D_{w}^{p}$, then $G \in H^{\infty}$ and $\|G\|_{\infty} \leq 1$ by Lemma 1.1 The isometric property of $G$ says that $\|f\|=\left\|G^{n} f\right\|$ for each $f \in D_{w}^{p}$ and every positive integer $n$. More explicitly,

$$
\|f\|_{D_{w}^{p}}^{p}=|G(0)|^{n p}|f(0)|^{p}+\int_{\mathbb{D})}\left|n G^{n-1} G^{\prime} f+G^{n} f^{\prime}\right|^{p} w d A .
$$

Suppose now that $G$ is not a unimodular constant. Then $|G(z)|<1$ everywhere in ID) by the maximum modulus principle, and $G(z)^{n} \rightarrow 0$ uniformly on compact subsets of $\mathbb{D D}$ as $n \rightarrow \infty$. In particular, $G(0)^{n} \rightarrow 0$ and

$$
\lim _{n \rightarrow \infty} \int_{\mathbb{D})}\left|G^{n} f^{\prime}\right|^{p} w d A=0,
$$

by the Lebesgue dominated convergence theorem. Hence by Minkowski's inequality,

$$
\|f\|_{D_{w}^{p}}^{p}=\lim _{n \rightarrow \infty} \int_{\mathbb{D} D}\left|n G^{n-1} G^{\prime} f\right|^{p} w d A
$$

for every polynomial $f$. If we apply (4.1) to the constant polynomial $f(z) \equiv 1$, we see that the total variations of the measures $d \mu_{n}=\left|n G^{n-1} G^{\prime}\right|^{p} w d A$ converge to 1 . Hence by Alaoglu's theorem, a subsequence $\left\{\mu_{n_{k}}\right\}$ converges in the weak-star topology to a probability measure $\mu$ on $\overline{\mathbb{D}}$. Using again the fact that $|G(z)|<1$ in $\mathbb{D}$, we see that $\mu$ must be supported on $T=\partial \mathbb{D}$ ). Consequently,

$$
\lim _{k \rightarrow \infty} \int_{\mathbb{D}} h\left|n_{k} G^{n_{k}-1} G^{\prime}\right|^{p} w d A=\int_{\mathbb{T}} h d \mu
$$

for every function $h$ continuous on $\overline{\mathbb{D}}$ ). Comparing this with (4.1), we conclude that

$$
|f(0)|^{p}+\int_{\mathbb{D}}\left|f^{\prime}\right|^{p} w d A=\int_{\mathbb{T}}|f|^{p} d \mu
$$

for every polynomial $f$.

We now apply (4.2) to the polynomials $f(z)=1+t z^{n}$, where $t$ is a real parameter, to obtain

$$
1+|t|^{p} n^{p} \int_{\mathbb{D}}|z|^{(n-1) p} w d A=\int_{\mathbb{T}}\left(1+2 t \Re\left\{z^{n}\right\}+t^{2}\right)^{p / 2} d \mu .
$$

Since the right-hand side of (4.3) is analytic in $t$ near 0 , we infer that $p$ must be an even integer. Also note that the coefficient of $t^{2}$ in the Taylor series development of the right-hand side is

$$
c_{2}=\int_{\mathbb{T}}\left(\frac{p}{2}+p(p-2)\left(\Re\left\{z^{n}\right\}\right)^{2}\right) d \mu,
$$


and $c_{2}>0$ because the integrand is strictly positive. On the other hand, if $p>2$, the left-hand side gives $c_{2}=0$, so the only possibility is that $p=2$. In this case, the coefficient of $t$ on the right-hand side of (4.3) must vanish; i.e.,

$$
\Re \int_{\mathbb{T}} z^{n} d \mu=0, \quad n=1,2, \ldots
$$

In a similar way, an application of (4.3) to the polynomials $f(z)=1+i t z^{n}$ gives

$$
\Im \int_{\mathbb{T}} z^{n} d \mu=0, \quad n=1,2, \ldots
$$

Since $\mu$ is real, it follows that

$$
\int_{\mathbb{T}} z^{n} d \mu=0, \quad n= \pm 1, \pm 2, \ldots
$$

and so, by the Weierstrass approximation theorem, $\mu$ must be normalized Lebesgue measure on the circle. Consequently, $D_{w}^{p}=D_{w}^{2}=H^{2}$, and the Littlewood-Paley formula shows that

$$
\int_{\mathbb{D})}\left|f^{\prime}(z)\right|^{2} w(z) d A(z)=2 \int_{\mathbb{D})}\left|f^{\prime}(z)\right|^{2} \log \frac{1}{|z|} d A(z)
$$

for all $f \in H^{2}$.

The final step is to deduce from (4.4) that $w(z)=-2 \log |z|$ a.e. in $\mathbb{D}$ ). Observe first that polarization identity gives the more general relation

$$
\int_{\mathbb{D}} f^{\prime}(z) \overline{g^{\prime}(z)} w(z) d A(z)=2 \int_{\mathbb{D})} f^{\prime}(z) \overline{g^{\prime}(z)} \log \frac{1}{|z|} d A(z)
$$

for all $f, g \in H^{2}$. In particular,

$$
\int_{\mathbb{D} D} z^{m} \bar{z}^{n} w(z) d A(z)=2 \int_{\mathbb{D})} z^{m} \bar{z}^{n} \log \frac{1}{|z|} d A(z)
$$

for all nonnegative integers $m$ and $n$. To apply (4.5), let $k \neq 0$ be any positive or negative integer, and choose nonnegative integers $n \geq-k$ and $m=n+k$. Then a calculation by polar coordinates gives

$$
\int_{0}^{1} r^{2 n+k+1} \int_{0}^{2 \pi} e^{i k \theta} w\left(r e^{i \theta}\right) d \theta d r=0
$$

since $m \neq n$ and the weight $-2 \log |z|$ is radial. If $n_{0}=\max \{-k, 0\}$, this means that the finite measure $\sigma$ on $[0,1]$ given by

$$
d \sigma=r^{2 n_{0}+k+1} \int_{0}^{2 \pi} e^{i k \theta} w\left(r e^{i \theta}\right) d \theta d r
$$


annihilates the monomials $r^{2 j}, j=0,1,2, \ldots$ Hence by the Weierstrass approximation theorem, $\sigma=0$. In other words,

$$
\int_{0}^{2 \pi} e^{i k \theta} w\left(r e^{i \theta}\right) d \theta=0 \quad k= \pm 1, \pm 2, \ldots
$$

for almost every $r \in[0,1]$. This implies that the weight function is radial, and we may write $w\left(r e^{i \theta}\right)=w(r)$ a.e. Setting $f^{\prime}(z)=z^{n}$ in (4.4), we have

$$
\int_{0}^{1} r^{2 n+1} w(r) d r=2 \int_{0}^{1} r^{2 n+1} \log \frac{1}{r} d r, \quad n=0,1,2, \ldots
$$

In other words, the measure

$$
d \nu=\left(w(r)-2 \log \frac{1}{r}\right) r d r
$$

annihilates the monomials $r^{2 j}$ for $j=0,1,2, \ldots$. Another appeal to the Weierstrass approximation theorem now shows that $\nu=0$, which completes the proof that $w(z)=-2 \log |z|$ a.e. in $\mathbb{D}$ ).

Remark It should be observed that Theorem 4.2 remains true for $0<p<1$. In fact, the same proof applies with one small modification. Instead of using Minkowski's inequality to obtain (4.1), one appeals to the inequality $\|f+g\|^{p} \leq\|f\|^{p}+\|g\|^{p}$, valid for $0<p<1$. Lemma 1.1 is stated for Banach spaces, but the proof extends readily to $D_{w}^{p}$ with $0<p<1$.

\section{Zero-divisors}

Let $X$ be a Banach space of analytic functions in the unit disk. A nonempty sequence $\left\{z_{k}\right\}$ of points in $\mathbb{D}$ ), finite or infinite, is called a zero-set for $X$ (or an $X$ zero-set) if some function $f \in X$ vanishes precisely on $\left\{z_{k}\right\}$. If all points $z_{k}$ are distinct, this means that $f\left(z_{k}\right)=0$ and $f^{\prime}\left(z_{k}\right) \neq 0$ for $k=1,2, \ldots$ If some point $\zeta$ occurs $m$ times in the sequence $\left\{z_{k}\right\}$, it means that $f$ has a zero of precise order $m$ at $\zeta$. An analytic function $f$ is said to vanish on $\left\{z_{k}\right\}$ if $f\left(z_{k}\right)=0$ for each $k$ and the order of its zero at $z_{k}$ is greater than or equal to the multiplicity of $z_{k}$ in the sequence. Such a function is allowed to vanish elsewhere in $\mathbb{D}$ ) and to have a zero of higher order than required at a point $z_{k}$.

A function $G \in X$ is called a zero-divisor for an $\mathrm{X}$ zero-set $\left\{z_{k}\right\}$ if it vanishes precisely on $\left\{z_{k}\right\}$ and $f / G \in X$ for every function $f \in X$ that vanishes on $\left\{z_{k}\right\}$. If $\|f / G\|=\|f\|$ for every such function $f$, then $G$ is said to be an isometric zero-divisor. Blaschke products are isometric zero-divisors for every Hardy space $H^{p}$. However, the next theorem will suggest that isometric zero-divisors are rather uncommon. Before stating the theorem, it is convenient to introduce the notion of a functional $\mathrm{Ba}$ nach space. This is a Banach space $X$ of functions analytic in a given domain $\Omega \subset \mathbb{C}$, with the properties:

(i) $X$ contains all polynomials; 
(ii) the polynomials are dense in $X$;

(iii) for $\zeta \in \Omega$, each point-evaluation functional $\phi_{\zeta}(f)=f(\zeta)$ is bounded in $X$.

Theorem 5.1 Let X be a functional Banach space, and suppose that $X$ is invariant under multiplication by polynomials. Then every isometric zero-divisor in $X$ is an isometric pointwise multiplier of $X$.

Proof Let $\left\{z_{k}\right\}$ be an $X$ zero-set and suppose $G \in X$ is an isometric zero-divisor for $\left\{z_{k}\right\}$. Then for each polynomial $Q$ the function $Q G$ belongs to $X$ and vanishes on $\left\{z_{k}\right\}$, so that

$$
\|Q\|=\left\|\frac{Q G}{G}\right\|=\|Q G\|
$$

We wish to conclude that $\|G f\|=\|f\|$ for every $f \in X$. To see this, let $\left\{Q_{n}\right\}$ be a sequence of polynomials for which $\left\|Q_{n}-f\right\| \rightarrow 0$. Such a sequence exists because the polynomials are assumed to be dense in $X$. Observe that $\left\{Q_{n} G\right\}$ is a Cauchy sequence in $X$, since $\left\|Q_{n} G-Q_{m} G\right\|=\left\|Q_{n}-Q_{m}\right\|$ by (5.1). Because $X$ is complete, it follows that $\left\|Q_{n} G-g\right\| \rightarrow 0$ for some function $g \in X$. By the assumption that point-evaluations are bounded, this implies that $Q_{n}(z) G(z) \rightarrow g(z)$ pointwise in ID). But for the same reason, $Q_{n}(z) \rightarrow f(z)$ pointwise, which shows that $g=f G$. Finally, in view of (5.1), the triangle inequality gives

$$
\begin{aligned}
|\|G f\|-\|f\|| & \leq\left|\|G f\|-\left\|Q_{n} G\right\|\right|+\left|\left\|Q_{n} G\right\|-\left\|Q_{n}\right\|\right|+\left|\left\|Q_{n}\right\|-\|f\|\right| \\
& \leq\left\|G f-Q_{n} G\right\|+\left\|Q_{n}-f\right\| \rightarrow 0 \text { as } n \rightarrow \infty .
\end{aligned}
$$

Thus $\|G f\|=\|f\|$ for every $f \in X$, as claimed.

In view of our results on isometric pointwise multipliers, it is now a short step to the following theorem.

\section{Theorem 5.2}

(i) For $1 \leq p<\infty$ and $\alpha>-1$, the Bergman spaces $A_{\alpha}^{p}$ with standard weights have no isometric zero-divisors. More generally, the weighted Bergman space $A_{w}^{p}$ has no isometric zero-divisors if it is a functional Banach space and $w(z)>0$ on some open subset of $\mathrm{D})$.

(ii) The Bloch space $\mathcal{B}$ and the little Bloch space $\mathcal{B}_{0}$ have no isometric zero-divisors.

(iii) The Dirichlet space $\mathcal{D}$ has no isometric zero-divisors. More generally, the Dirichlettype space $D_{w}^{p}$ has no isometric zero-divisors if it is a functional Banach space, except for $p=2$ and $w(z)=-2 \log |z|$, where $D_{w}^{p}=H^{2}$.

(iv) For $1 \leq p<\infty$, the only isometric zero-divisors of the Hardy space $H^{p}$ are the Blaschke products.

Proof (i) As mentioned earlier, polynomials are dense and point-evaluation functionals are bounded in the standard weighted Bergman spaces $A_{\alpha}^{p}$, so they are functional Banach spaces. If more generally $A_{w}^{p}$ is a functional Banach space, then by Theorem 5.1 any isometric zero-divisor would be an isometric pointwise multiplier. But under the additional hypothesis that $w(z)>0$ on an open set, Theorem 2.1 says 
that the only isometric pointwise multipliers of $A_{w}^{p}$ are the unimodular constants, which are not zero-divisors.

(ii) The polynomials are dense in $\mathcal{B}_{0}$, so it is a functional Banach space. It is easy to check that $\mathcal{B}_{0}$ is invariant under multiplication by polynomials. Hence by Theorem 5.1 every isometric zero-divisor of $\mathcal{B}_{0}$ is an isometric pointwise multiplier. But by Theorem 3.1 the only isometric pointwise multipliers of $\mathcal{B}_{0}$ are the unimodular constants. Thus $\mathcal{B}_{0}$ has no isometric zero-divisors. Since $\mathcal{B}_{0} \subset \mathcal{B}$, this clearly shows that no function $G \in \mathcal{B}_{0}$ is an isometric zero-divisor of $\mathcal{B}$, but it leaves open the possibility that some function in $\mathcal{B} \backslash \mathcal{B}_{0}$ may be. The difficulty is surmounted by appeal to the following lemma.

Lemma 5.3 Every isometric zero-divisor of $\mathcal{B}$ belongs to $\mathcal{B}_{0}$.

The proof of Lemma5.3 is deferred to the end of this section.

(iii) This follows by combining Theorem 5.1 with Theorem 4.1 and its generalization, Theorem 4.2 .

(iv) By Theorem 5.1 and Theorem 2.1, every zero-divisor of $H^{p}$ is an inner function. However, the canonical factorization of $H^{p}$ functions (see [5]) makes clear that an inner function cannot be a divisor of the space if it has a nontrivial singular factor. Hence the zero-divisors are precisely the Blaschke products.

Proof of Lemma 5.3 Let $G$ be an isometric zero-divisor of $\mathcal{B}$. For $a \in \mathbb{D}$ ), the functions $f_{a}(z)=(1-\bar{a} z)^{-1}$ and $g_{a}(z)=-\log (1-\bar{a} z)$ are pointwise multipliers of $\mathcal{B}$, since they are analytic in the closed disk. It follows as in (5.1) that

$$
\left\|G f_{a}\right\|_{\mathcal{B}}=\left\|f_{a}\right\|_{\mathcal{B}} \quad \text { and } \quad\left\|G g_{a}\right\|_{\mathcal{B}}=\left\|g_{a}\right\|_{\mathcal{B}} .
$$

The first equality gives

$$
\begin{gathered}
|G(1)|+\left(1-|a|^{2}\right)\left|\left(G f_{a}\right)^{\prime}(a)\right| \leq\left\|f_{a}\right\|_{\mathcal{B}}=1+\frac{|a|}{1-|a|^{2}}, \quad \text { or } \\
\left.\left|\bar{a} G(a)+\left(1-|a|^{2}\right) G^{\prime}(a)\right| \leq|a|+\left(1-|a|^{2}\right)(1-|G(1)|), \quad a \in \mathbb{D}\right),
\end{gathered}
$$

and since $G \in \mathcal{B}$, we conclude that $G$ is bounded. The equality $\left\|G g_{a}\right\|_{\mathcal{B}}=\left\|g_{a}\right\|_{\mathcal{B}}$ implies

$$
\begin{gathered}
\left(1-|a|^{2}\right)\left|\left(G g_{a}\right)^{\prime}(a)\right| \leq\left\|g_{a}\right\|_{\mathcal{B}}<2, \quad \text { or } \\
\left.\left|\bar{a} G(a)+\left(1-|a|^{2}\right) G^{\prime}(a) \log \left(1-|a|^{2}\right)\right|<2, \quad a \in \mathbb{D}\right) .
\end{gathered}
$$

Since $G$ is bounded, we conclude that $G \in \mathcal{B}_{0}$.

Lemma 5.3 and its proof are closely related to the description of pointwise multipliers of the Bloch space, as given by Brown and Shields [1]. They showed that an analytic function $G$ is a multiplier of $\mathcal{B}$ if and only if it is a multiplier of $\mathcal{B}_{0}$, and these multipliers are precisely the functions $G \in H^{\infty}$ with the property

$$
G^{\prime}(z)=O\left(\frac{1}{(1-|z|) \log (1 /(1-|z|))}\right), \quad|z| \rightarrow 1 .
$$




\section{Coefficient Multipliers}

Following our discussion of isometric pointwise multipliers, it is natural to ask for a description of isometric coefficient multipliers. In terms of a complex sequence $\Lambda=\left\{\lambda_{n}\right\}$, the coefficient multiplier operator $M_{\Lambda}$ is defined formally on functions $f$ analytic in the unit disk by

$$
\left(M_{\Lambda} f\right)(z)=\sum_{n=0}^{\infty} \lambda_{n} a_{n} z^{n}, \quad \text { where } f(z)=\sum_{n=0}^{\infty} a_{n} z^{n}
$$

If $X$ and $Y$ are spaces of analytic functions in $\mathbb{D}$ ), the sequence $\Lambda$ is called a coefficient multiplier of $X$ into $Y$ if $M_{\Lambda}$ maps $X$ into $Y$. It is an isometric coefficient multiplier of $X$ into $Y$ if $\left\|M_{\Lambda} f\right\|_{Y}=\|f\|_{X}$ for all $f \in X$.

It is easy to see that $\Lambda$ is a multiplier of the Hardy space $H^{2}$ into itself, or of the Bergman space $A^{2}$ into itself, if and only if it is a bounded sequence. It is an isometric multiplier if and only if $\left|\lambda_{n}\right|=1$ for all $n$. For $p \neq 2$, however, the situation is quite different. Then the spaces $H^{p}$ and $A^{p}$ are not solid: they need not be preserved under multiplication by a bounded sequence $\Lambda$. (See [2] for a full discussion of this phenomenon.) On the other hand, rotations are isometries that can be described by coefficient multipliers. The following theorem says that the given spaces have no other isometric coefficient multipliers.

Theorem 6.1 For $1 \leq p<\infty$, with $p \neq 2$, the only isometric coefficient multipliers of the Hardy space $H^{p}$ or the standard weighted Bergman space $A_{\alpha}^{p}$ are those of the form $\Lambda=\left\{c \lambda^{n}\right\}$, where $|c|=|\lambda|=1$. The same is true for the Bloch space $\mathcal{B}$ and the little Bloch space $\mathcal{B}_{0}$.

The proof will use the following descriptions of the surjective isometries of Hardy spaces, Bergman spaces, and the Bloch space, due respectively to Forelli [8], Kolaski [10], and Cima and Wogen [3].

Theorem $A$ Let $1 \leq p<\infty$ and $p \neq 2$. Then every linear surjective isometry $T: H^{p} \rightarrow H^{p}$ has the form

$$
T f=a(f \circ \varphi)\left(\varphi^{\prime}\right)^{1 / p}, \quad f \in H^{p}
$$

for some conformal automorphism $\varphi: \mathbb{D}) \rightarrow \mathbb{D})$ and some unimodular constant a.

Theorem $B$ Let $1 \leq p<\infty, p \neq 2$, and $\alpha>-1$. Then every linear surjective isometry $T: A_{\alpha}^{p} \rightarrow A_{\alpha}^{p}$ has the form

$$
T f=a(f \circ \varphi)\left(\varphi^{\prime}\right)^{(2+\alpha) / p}, \quad f \in A_{\alpha}^{p}
$$

for some conformal automorphism $\varphi: \mathbb{D}) \rightarrow \mathbb{D}$ ) and some unimodular constant a.

It may be noted that the spaces $H^{2}$ and $A_{\alpha}^{2}$ are Hilbert spaces and so have many surjective isometries. 
Theorem $C$ Every linear surjective isometry $T: \mathcal{B} \rightarrow \mathcal{B}$ has the form

$$
T f=a(f \circ \varphi-f(\varphi(0))+f(0)), \quad f \in \mathcal{B}_{0}
$$

for some conformal automorphism $\varphi: \mathbb{D}) \rightarrow \mathbb{D}$ ) and some unimodular constant a. Moreover, every linear isometry of $\mathcal{B}_{0}$ has the same form and is therefore surjective.

Note that the action of surjective isometries of $\mathcal{B}$ is described only on the subspace $\mathcal{B}_{0}$. This will not affect our proof, however, since $\mathcal{B}_{0}$ contains the polynomials.

Proof of Theorem 6.1 The strategy is to show that whenever a coefficient multiplier is isometric, it is automatically surjective, so that Theorems $\mathrm{A}$ and $\mathrm{B}$ apply. We will carry out the proof only for the space $A_{\alpha}^{p}$, as the discussions for $H^{p}$ and $\mathcal{B}$ are essentially the same. If an analytic function $f(z)=\sum_{n=0}^{\infty} a_{n} z^{n}$ belongs to $A_{\alpha}^{p}$, then so does the function $f^{*}(z)=\overline{f(\bar{z})}=\sum_{n=0}^{\infty} \overline{a_{n}} z^{n}$, and the two functions have equal norms. Hence it is easy to see that if $\Lambda$ is a coefficient multiplier of $A_{\alpha}^{p}$ into itself, then so is $\bar{\Lambda}=\left\{\overline{\lambda_{n}}\right\}$. Moreover, $\bar{\Lambda}$ is an isometric multiplier if $\Lambda$ is. But if $\Lambda$ is an isometric multiplier of $A_{\alpha}^{p}$, then by applying it to the monomials $z^{n}$ we readily see that $\left|\lambda_{n}\right|=1$ for all $n$. This shows that the identity operator on $A_{\alpha}^{p}$ satisfies $I=M_{\Lambda} M_{\bar{\Lambda}}$. Hence $M_{\Lambda}$ is surjective, and is therefore a weighted composition operator of the form specified in Theorem B. Applying this to the identity function $f(z)=z$, we obtain

$$
\lambda_{1} z=a \varphi(z)\left(\varphi^{\prime}(z)\right)^{(2+\alpha) / p}
$$

which shows that the conformal automorphism $\varphi$ must be a rotation: $\varphi(z)=\lambda z$ for $|\lambda|=1$. Therefore,

$$
\left(M_{\Lambda} f\right)(z)=c f(\lambda z)=c \sum_{n=0}^{\infty} a_{n} \lambda^{n} z^{n}
$$

where $|c|=1$, as claimed.

As a simple consequence, we find that the result holds also for the Dirichlet-type spaces $D_{\alpha}^{p}=D_{w_{\alpha}}^{p}$ with standard radial weights $w_{\alpha}$.

Corollary For $1 \leq p<\infty, p \neq 2$, and $\alpha>-1$, the only isometric coefficient multipliers of the Dirichlet-type space $D_{\alpha}^{p}$ are those of the form $\Lambda=\left\{c \lambda^{n}\right\}$, where $|c|=|\lambda|=1$.

In closing, it may be remarked that the isometric composition operators of the Bloch space, the Dirichlet space, and various other spaces were recently described in $[4,11-13]$. As a common generalization of those results and the ones in the current paper, it would be interesting to find a description of the isometries among the weighted composition operators. 


\section{References}

[1] L. Brown and A. L. Shields, Multipliers and cyclic vectors in the Bloch space. Michigan Math. J. 38(1991), 141-146. doi:10.1307/mmj/1029004269

[2] S. M. Buckley, P. Koskela and D. Vukotić, Fractional integration, differentiation, and weighted Bergman spaces. Math. Proc. Cambridge Philos. Soc. 126(1999), 369-385. doi:10.1017/S030500419800334X

[3] J. A. Cima and W. Wogen, On isometries of the Bloch space. Illinois J. Math. 24(1980), 313-316.

[4] F. Colonna, Characterisation of the isometric composition operators on the Bloch space. Bull. Austral. Math. Soc. 72(2005), 283-290. doi:10.1017/S0004972700035073

[5] P. L. Duren, Theory of $H^{p}$ Spaces. Academic Press, New York, 1970; reprinted with supplement by Dover Publications, Mineola, NY, 2000.

[6] P. Duren and A. Schuster, Bergman Spaces. American Mathematical Society, Providence, RI, 2004.

[7] P. Duren, D. Khavinson, H. S. Shapiro and C. Sundberg, Contractive zero-divisors in Bergman spaces. Pacific J. Math. 157(1993), 37-56.

[8] F. Forelli, The isometries of $H^{p}$. Canad. J. Math. 16(1964), 721-728.

[9] H. Hedenmalm, A factorization theorem for square area-integrable analytic functions. J. Reine Angew. Math. 422(1991), 45-68.

[10] C. J. Kolaski, Isometries of weighted Bergman spaces. Canad. J. Math. 34(1982), 910-915.

[11] M. J. Martín and D. Vukotić, Isometries of the Dirichlet space among the composition operators. Proc. Amer. Math. Soc. 134(2006), 1701-1705. doi:10.1090/S0002-9939-05-08182-7

[12] M. J. Martín and D. Vukotić, Isometries of some classical function spaces among the composition operators. Contemp. Math. 393(2006), 133-138.

[13] Isometries of the Bloch space among the composition operators. Bull. London Math. Soc. 39(2007), 151-155.

[14] D. Stegenga, Multipliers of the Dirichlet space. Illinois J. Math. 24(1980), 113-139.

Department of Mathematics, Lund University, P. O. Box 118, S-221 00 Lund, Sweden e-mail: aleman@maths.lth.se

Department of Mathematics, University of Michigan, Ann Arbor, Michigan 48109-1043, USA

e-mail: duren@umich.edu

Departamento de Matemáticas, Universidad Autónoma de Madrid, 28049 Madrid, Spain

e-mail: mariaj.martin@uam.es

Departamento de Matemáticas \& ICMAT CSIC-UAM-UC3M-UCM, Universidad Autónoma de Madrid, 28049 Madrid, Spain

e-mail: dragan.vukotic@uam.es 